ARTigo OriginAL

Original Article

\title{
Estudo sobre as Diferentes Formas Clínicas e Escores Cutâneos na Esclerose Sistêmica ${ }^{(*)}$
}

\author{
Study about Different Clinical Variants and \\ Skin Scores in Systemic Sclerosis
}

\author{
Percival D. Sampaio-Barros ${ }^{(1)}$, Adil Muhib Samara ${ }^{(2)}$ e João Francisco Marques Neto ${ }^{(2)}$
}

\section{RESUMO}

Objetivo: Descrever a experiência com o uso do escore cutâneo total (ECT), utilizando-se os métodos de Rodnan original (ECTRO) e modificado (ECT-RM) na esclerose sistêmica (ES), analisando seu significado clínico nos modelos de duas (difusa e limitada) e três (difusa, intermediária e limitada) formas clínicas. Métodos: Estudo prospectivo analisando o espessamento cutâneo (obtido através do ECT pelos métodos de Rodnan original e modificado) em 56 pacientes com ES. Os resultados do ECT foram comparados com as formas clínicas da ES (nos modelos de duas e três formas clínicas), bem como com manifestações cutâneas (calcinose, telangiectasias, distúrbios de pigmentação), sistêmicas (articular, vascular, esofágica, pulmonar, cardíaca, renal) e alterações laboratoriais (fator antinuclear, anticorpo anticentrômero, anticorpo anti-topoisomerase I). Os resultados do ECT também foram subdivididos em ECT baixo $(\leq 20)$ e ECT elevado $(>20)$, em ambos os métodos. Análise de regressão logística foi realizada para se determinar quais as variáveis independentes que mais influenciaram ECT e óbito (após cinco anos de seguimento). Resultados: Houve associação estatística entre ECT $\leq 20$ e ES limitada (ambos os métodos de ECT no modelo com duas formas clínicas), calcinose (ECT-RM) e anticorpo anticentrômero (ECT-RO, tendência). ECT $>20$ esteve associado com ES difusa (ambos os métodos de ECT nos modelos de duas e três formas clínicas), distúrbios de pigmentação (ambos os métodos de ECT), acometimento articular (ambos os métodos de ECT), doença pulmonar restritiva (ECT-RM, tendência) e anticorpo anti-topoisomerase I (ECT-RO, tendência). Análise de regressão logística multivariada indicou que a forma clínica foi a variável que influenciou de maneira mais significativa o ECT (em ambos os métodos de ECT). Após seguimento de cinco anos, ocorreram 20 óbitos (35.7\%);

\begin{abstract}
Objective: To report the experience using the Total Skin Score (TSS), in the original (OR-TSS) and modified (MR-TSS) Rodnan methods in systemic sclerosis (SSc), analysing its clinical significance in the model of two (diffuse and limited) and three (diffuse, intermediate and limited) clinical variants. Methods: Prospective study analysing skin thickness (assessed by original and modified Rodnan TSS methods) in 56 SSC patients. TSS results were compared with SSc clinical variants (subdivided in the model of two and three subgroups) as well as with cutaneous (calcinosis, telangiectasias, pigmentary abnormalities), systemic (articular, vascular, esophageal, pulmonary, cardiac, renal) and laboratory (antinuclear antibody, anticentromere antibody, anti-topoisomerase I antibody) manifestations. Data were analysed based on the presence of a low $(\leq 20)$ or a high TSS $(>20)$ by both methods. Logistic regression analysis was performed to determine the independent variables that influenced TSS (in or by both methods) and death (after a 5-year follow-up). Results: There was statistical association between TSS $\leq 20$ and limited SSC (both methods in the SSc model of two subgroups), calcinosis (MR-TSS) and anticentromere antibody (OR-TSS, trend). TSS $>20$ was associated with diffuse SSc (both methods in the SSc models of two and three subgroups), pigmentary abnormalities (both methods), articular involvement (both methods), pulmonary restrictive disease (MR-TSS, trend) and anti-topoisomerase I antibody (OR-TSS, trend). Multivariate logistic regression analysis indicated that the SSc clinical variant was the most important factor that influenced significantly TSS (in both methods). After a 5-year follow-up, there were
\end{abstract}

\footnotetext{
* Trabalho realizado na Disciplina de Reumatologia do Departamento de Clínica Médica da Faculdade de Ciências Médicas da Universidade Estadual de Campinas (FCM-UNICAMP), Campinas - SP. Este trabalho contou com o apoio do Fundo de Amparo à Pesquisa da Sociedade Brasileira de Reumatologia. Trabalho recebido em 29/09/03. Aprovado, após revisão, em 12/01/04.

1. Assistente-Doutor. (FCM-UNICAMP).

2. Professor Titular. (FCM-UNICAMP).
}

Endereşo para correspondência: Dr. Percival D. Sampaio-Barros. Disciplina de Reumatologia, Departamento de Clínica Médica. Faculdade de Ciências Médicas Universidade Estadual de Campinas (UNICAMP). Barão Geraldo, Campinas, SP, CEP 13081-970. E-mail: psbarros@fcm.unicamp.br 
análise de regressão logística multivariada indicou que o acometimento vascular foi a variável que mais influenciou o óbito, independente do ECT. Conclusões: O ECT, nos métodos de Rodnan original e modificado, permitiu diferenciar distintos padrões de doença no espectro clínico da ES. No modelo de duas formas clínicas existem claras distinções entre a ES limitada e a ES difusa, enquanto que no modelo de três formas clínicas esta distinção não é tão claramente observada.

Palavras-chave: esclerose sistêmica, escore cutâneo, espessamento cutâneo.

\section{INTRODUÇÃO}

O espessamento cutâneo representa o principal marcador clínico da esclerose sistêmica $(\mathrm{ES})^{(1)}$, base para a classificação da doença em diferentes formas clínicas ${ }^{(2)}$. Em 1971, dois estudos independentes sugeriram que o acometimento cutâneo da pele do tronco estava associado a um pior prognóstico na $\mathrm{ES}^{(3,4)}$. Durante as duas décadas seguintes, diferentes modelos de classificação da ES foram sendo propostos, resultando no reconhecimento de duas ${ }^{(2)}$ ou três ${ }^{(5-7)}$ formas clínicas, de acordo com a extensão do acometimento cutâneo. Estes estudos enfatizaram que a forma clínica "difusa" apresentava um pior prognóstico que as formas clínicas "limitada" ou "intermediária".

Vários métodos para a aferição do espessamento cutâneo na ES têm sido propostos, incluindo diversos aparelhos para avaliar a elasticidade cutânea ${ }^{(8-10)}$ a ultra-sonografia da pele ${ }^{(11-13)}$. Atualmente, o método mais utilizado para a avaliacão periódica do espessamento cutâneo na ES tem sido o escore cutâneo ${ }^{(14-17)}$. Rodnan foi o primeiro a introduzir um método semiquantitativo para a determinacão da extensão e da gravidade do acometimento cutâneo na ES, o Escore Cutâneo Total $(\mathrm{ECT})^{(14)}$. Foi proposta uma possível relação entre o método ECT original de Rodnan e a sobrevida: pacientes com ECT $>40$ apresentaram um índice de sobrevida de 50\% em 5 anos, enquanto aqueles com ECT $\leq 40$ apresentaram um índice de sobrevida de 70\% no mesmo período $^{(18)}$. Na última década, várias modificacões foram propostas no método ECT original de Rodnan ${ }^{(19-24)}$, a fim de reduzir a variabilidade inter e intraobservador.

No presente estudo, o ECT (avaliado nos métodos de Rodnan original e modificado) foi aplicado a uma população brasileira de pacientes com ES, e os resultados foram comparados com variáveis clínicas (divididas nos modelos de duas e três formas clínicas), cutâneas, sistêmicas e laboratoriais, e evolução para óbito (após seguimento de cinco anos).
20 deaths (35.7\%); multivariate logistic regression analysis indicated that vascular involvement influenced death significantly, despite the TSS score. Conclusions: TSS, by both original and modified Rodnan methods, permits to differentiate distinct patterns in the clinical spectrum of SSC. In the model of two subgroups, there is a clear distinction between diffuse and limited disease, whereas in the model of three subgroups this distinction is not so clearly observed between the variants.

Keywords: systemic sclerosis, skin score, skin thickness.

\section{$\overline{\text { MATERIAL E MÉTODOS }}$}

Pacientes: Este estudo prospectivo avaliou 56 pacientes com diagnóstico de ES que preenchiam os critérios classificatórios do Colégio Americano de Reumatologia ${ }^{(1)}$, acompanhados no ambulatório de Esclerodermia do Hospital de Clínicas da Unicamp. No modelo de duas formas clínicas, os pacientes foram subdivididos em ES difusa e ES limitada, de acordo com os critérios de LeRoy e colaboradores $^{(2)}$. No modelo de três formas clínicas, os pacientes foram subdivididos em ES difusa, ES intermediária e ES limitada de acordo com os seguintes critérios: limitada esclerodactilia isolada; intermediária - espessamento cutâneo proximal às articulações metacarpofalangeanas, poupando o tronco; difusa - espessamento difuso da pele incluindo o tronco. Pacientes com esclerodermia localizada foram excluídos do estudo.

O grupo estudado incluiu no modelo de ES com duas formas clínicas, 36 pacientes com ES limitada e 20 pacientes com ES difusa, enquanto que, no modelo com três formas clínicas, 10 pacientes com ES limitada, 34 pacientes com ES intermediária e 12 pacientes com ES difusa.

Desenho do estudo: No início do estudo, todos os pacientes foram avaliados segundo um protocolo padrão de investigação. A extensão e a gravidade do espessamento cutâneo foram avaliados pelo ECT, pelos métodos de Rodnan original e modificado. O ECT original de Rodnan (ECT-RO) é definido como a soma da estimativa do grau de espessamento cutâneo, pela palpação, de 26 sítios anatômicos $^{(14)}$ (Figura 1). A estimativa do espessamento cutâneo foi graduada segundo a seguinte escala: $0=$ pele normal; 1 = espessamento leve mas definido; 2 = espessamento leve a moderado; 3 = espessamento intenso; 4 = grau extremo de espessamento. O ECT-RO pode variar entre 0 e 104. 


\begin{tabular}{|c|c|c|c|c|c|c|c|c|c|c|c|c|c|}
\hline \multicolumn{8}{|c|}{ DIREITA } & \multicolumn{6}{|c|}{ ESQUERDA } \\
\hline 0 & 1 & 2 & 3 & 4 & DEDOS & & & & 0 & 1 & 2 & 3 & 4 \\
\hline 0 & 1 & 2 & 3 & 4 & ORSO MÃO & & & & 0 & 1 & 2 & 3 & 4 \\
\hline 0 & 1 & 2 & 3 & 4 & ANTEBRAÇO & & & & 0 & 1 & 2 & 3 & 4 \\
\hline 0 & 1 & 2 & 3 & 4 & BRAÇOS & & & & 0 & 1 & 2 & 3 & 4 \\
\hline \multirow[t]{4}{*}{0} & 1 & 2 & 3 & 4 & OMBROS & & & & 0 & 1 & 2 & 3 & 4 \\
\hline & & & & FACE & 0 & 1 & 2 & 3 & 4 & & & & \\
\hline & & & & PESCOÇO & 0 & 1 & 2 & 3 & 4 & & & & \\
\hline & & & & TÓRAX & 0 & 1 & 2 & 3 & 4 & & & & \\
\hline \multirow[t]{4}{*}{0} & 1 & 2 & 3 & 4 & MAMAS & & & & 0 & 1 & 2 & 3 & 4 \\
\hline & & & & ABDOMEN & 0 & 1 & 2 & 3 & 4 & & & & \\
\hline & & & & DORSAL & 0 & 1 & 2 & 3 & 4 & & & & \\
\hline & & & & LOMBAR & 0 & 1 & 2 & 3 & 4 & & & & \\
\hline 0 & 1 & 2 & 3 & 4 & COXAS & & & & 0 & 1 & 2 & 3 & 4 \\
\hline 0 & 1 & 2 & 3 & 4 & PERNAS & & & & 0 & 1 & 2 & 3 & 4 \\
\hline 0 & 1 & 2 & 3 & 4 & DORSO PÉS & & & & 0 & 1 & 2 & 3 & 4 \\
\hline 0 & 1 & 2 & 3 & 4 & DEDOS & & & & 0 & 1 & 2 & 3 & 4 \\
\hline
\end{tabular}

Figura 1 - Escore Cutâneo Total - Método de Rodnan Original (ECT-R0).

O ECT modificado de Rodnan (ECT-RM) $)^{(19-24)}$ consiste na avaliação do espessamento da pele, pela palpação, em 17 sítios anatômicos (Figura 2), retirados do ECT original de Rodnan. A estimativa do espessamento cutâneo foi graduada utilizando uma escala de 0 a 3 , sendo $0=$ pele normal, 1 = espessamento cutâneo leve, 2 = espessamento cutâneo moderado, 3 = espessamento cutâneo intenso, com incapacidade de preguear a pele. O ECT-RM pode variar entre 0 e 51 . As avaliações do ECT, pelos métodos de Rodnan original e modificado, foram realizadas na visita inicial do paciente no ambulatório de Esclerodermia, pelo mesmo observador.

Outras manifestacões cutâneas, como calcinose, telangiectasias e distúrbios de pigmentação também foram investigadas.

As seguintes definições foram utilizadas a fim de descrever acometimento orgânico específico, na avaliação inicial do estudo: 1) Articular: artrite ou poliartralgia periférica; 2) Vascular: ulceração de polpas digitais ou reabsorção de falanges distais ou necrose extensa e/ou amputação; 3) Esôfago: disfagia, associada à evidência radiológica de hipomotilidade do esôfago distal; 4) Intestinos: hábito intestinal alterado, associado ao distúrbio de motilidade nos

\begin{tabular}{|c|c|c|c|c|c|c|c|c|c|c|c|}
\hline \multicolumn{4}{|c|}{ DIREITA } & & & & & \multicolumn{4}{|c|}{ ESQUERDA } \\
\hline 0 & 1 & 2 & 3 & & DEDOS & & & 0 & 1 & 2 & 3 \\
\hline 0 & 1 & 2 & 3 & & ORSO MÃOS & & & 0 & 1 & 2 & 3 \\
\hline 0 & 1 & 2 & 3 & & NTEBRAÇO & & & 0 & 1 & 2 & 3 \\
\hline \multirow[t]{4}{*}{0} & 1 & 2 & 3 & & BRAÇOS & & & 0 & 1 & 2 & 3 \\
\hline & & & & FACE & 0 & 1 & 2 & 3 & & & \\
\hline & & & & TÓRAX & 0 & 1 & 2 & 3 & & & \\
\hline & & & & ABDOMEN & 01 & 1 & 2 & 3 & & & \\
\hline 0 & 1 & 2 & 3 & & COXAS & & & 0 & 1 & 2 & 3 \\
\hline 0 & 1 & 2 & 3 & & PERNAS & & & 0 & 1 & 2 & 3 \\
\hline 0 & 1 & 2 & 3 & & DORSO PÉS & & & 0 & 1 & 2 & 3 \\
\hline
\end{tabular}

FiguRa 2 - Escore CutâneoTotal - Método de Rodnan Modificado (ECT-RM).

exames radiológicos contrastados de intestino delgado e/ ou grosso; 5) Doença pulmonar restritiva (DPR): dispnéia aos esforços, associada à evidência de fibrose intersticial em bases pulmonares à radiografia de tórax e/ou defeito ventilatório restritivo (capacidade vital forçada $<70 \%$ do esperado) à prova de função pulmonar; 6) Hipertensão pulmonar: aumento da pressão sistólica da artéria pulmonar $(\geq 30 \mathrm{mmHg}$ ), documentado pelo ecocardiograma com doppler, isolado ou associado à DPR; 7) Coração: insuficiência cardíaca congestiva ou pericardite ou arritmia sintomáticas; 8) Rins: crise renal esclerodérmica, caracterizada por insuficiência renal rapidamente progressiva associada à hipertensão arterial maligna.

A pesquisa de auto-anticorpos séricos foi realizada na avaliação inicial dos pacientes. Foram utilizados os seguintes métodos: fator antinuclear (FAN) e anticorpo anticentrômero, utilizando-se imunofluorescência indireta em células HEp-2 como substrato; anticorpo anti-topoisomerase I (anti-Scl 70), usando a técnica de micro-hemaglutinação. Os resultados foram considerados positivos quando a fluorescência esteve positiva a partir do título 1:40.

Os resultados do ECT (pelos métodos de Rodnan original e modificado) foram subdivididos em ECT baixo $(\leq 20)$ e ECT elevado (>20), sendo correlacionados com as formas clínicas da ES e a presença de manifestações cutâneas, sistêmicas e laboratoriais ao início do estudo, bem como com a evolução para óbito (após cinco anos de seguimento).

Análise estatística: Os dados foram analisados, nos casos de associação, utilizando-se o teste do qui-quadrado $\left(x^{2}\right)$ e o teste exato de Fisher. Testes não-paramétricos de MannWhitney foram utilizados para comparar dados quanti- 
tativos. Evidência de significância estatística requereu valores de $p \leq 0.05$; tendência estatística foi considerada quando $0.05<p \leq 0.10$.

Análises de regressão logística, univariada e multivariada, foram utilizadas para determinar quais variáveis independentes influenciaram o ECT (em ambos os métodos) e o óbito (após cinco anos de seguimento).

\section{RESULTADOS}

No modelo de ES com duas formas clínicas, o ECT-RO médio foi de $12.9 \pm 6.1$ (mediana 11.5) para a ES limitada e $34.2 \pm 11.2$ (mediana 32 ) para a ES difusa, enquanto que o ECT-RM médio foi de $11.8 \pm 4.9$ (mediana 11.5) para a ES limitada e $26.2 \pm 5.8$ (mediana 25) para a ES difusa. Foi observada associação estatística entre ECT $\leq 20$ e ES limitada e entre ECT $>20$ e ES difusa, nos dois métodos de ECT $(p=0.001)$ (Figuras 3 e 4).

No modelo de ES com três formas clínicas, o ECT-RO médio foi de $6.9 \pm 2.8$ (mediana 8) para a ES limitada, 18 \pm 7.5 (mediana 18) para a ES intermediária e $38.8 \pm 11.7$ (mediana 36) para a ES difusa, enquanto que o ECT-RM médio foi de $6.9 \pm 2.8$ (mediana 8) para a ES limitada, $15.9 \pm 5.6$ (mediana 16) para a ES intermediária e $28.3 \pm$ 6.2 (mediana 26) para a ES difusa. Foi observada associação estatística entre ECT $>20$ e ES difusa, nos dois métodos de ECT $(p=0.001)$ (Figuras 5 e 6$)$.

Com respeito às manifestações cutâneas, 25 pacientes (44.6\%) apresentaram calcinose, 34 (60.7\%) telangiectasias e 33 (58.9\%) distúrbios de pigmentação (localizado ou difuso). Foram observadas as seguintes manifestações sistêmicas: articular em 33 pacientes (58.9\%), vascular em 20 (35.7\%), esofágica em 38 (67.9\%), intestinal em dois (3.6\%), DPR em 30 (53.6\%), hipertensão pulmonar em 20 (35.7\%), cardíaca em oito (14.3\%) e renal em dois pacientes (3.6\%). O FAN foi positivo em 46 pacientes (82.1\%), enquanto que o anticorpo anticentrômero foi positivo em 10 (17.6\%) e o anti-topoisomerase I em 14 pacientes (25\%).

No modelo de ES com duas formas clínicas, a ES difusa esteve associada ao ECT $>20$ (ambos os métodos de ECT; $p=0.001)$ e aos distúrbios de pigmentação $(p=0.001)$, com tendência estatística relacionada à DPR $(p=0.066) \mathrm{e}$ ao anticorpo anti-topoisomerase I $(p=0.053)$, enquanto que a ES limitada esteve associada ao ECT $\leq 20(p=0.001)$, à calcinose $(p=0.001)$ e ao anticorpo anticentrômero $(p=0.009)$. No modelo de ES com três formas clínicas, a ES difusa esteve associada com o ECT $>20$ (ambos os métodos de ECT; $p=0.001)$ e distúrbios de pigmentação $(p=0.001)$, a ES intermediária esteve associada à calcinose $(p=0.001)$ e às telangiectasias $(p=0.001)$, enquanto que a ES limitada esteve negativamente associada ao acometimento articular $(p=0.001)$ e vascular $(p=0.015)$.

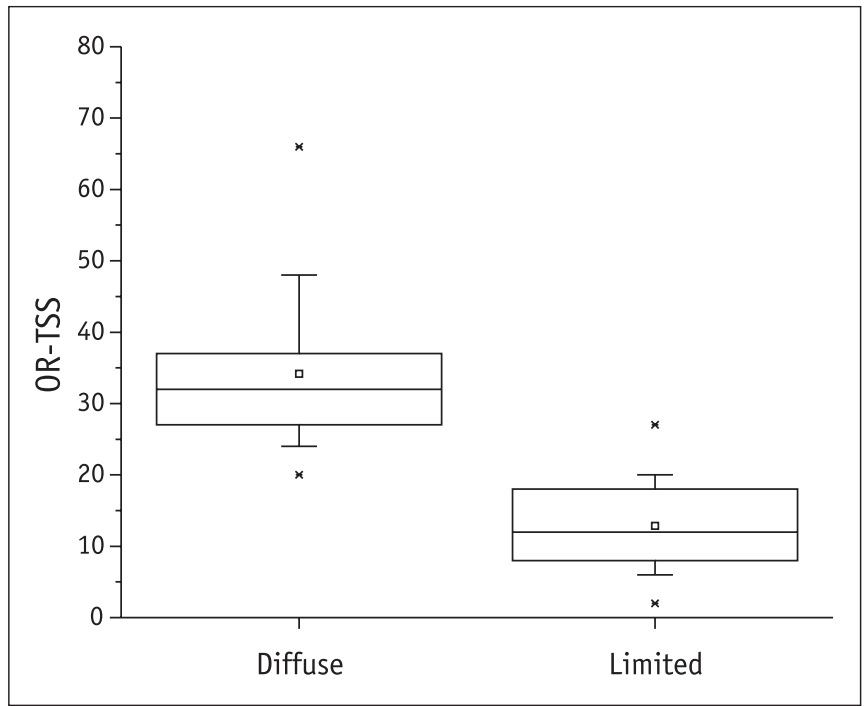

FiguRa 3 - Escore Cutâneo Total - Método de Rodnan Original (ECT-R0); modelo de duas formas clínicas: difusa $(n=20)$ e limitada $(n=36)$; ${ }^{*} p<0,0001$ (teste Mann-Whitney).

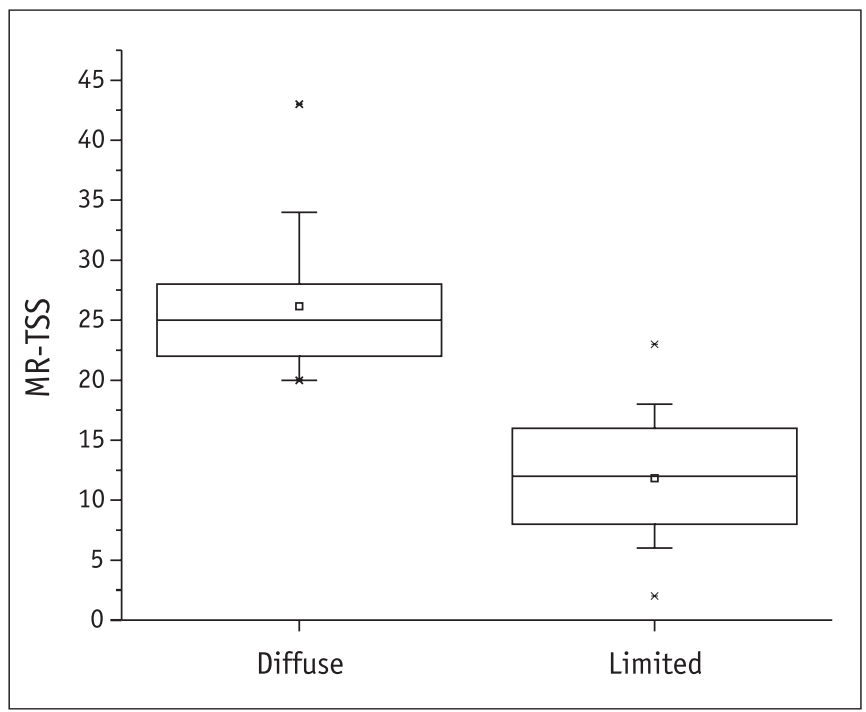

FIGURA 4- Escore Cutâneo Total - Método de Rodnan Modificado (ECT-RM); modelo de duas formas clínicas: difusa $(n=20)$ e limitada $(n=36)$; ${ }^{*} p<0,0001$ (teste Mann-Whitney). 


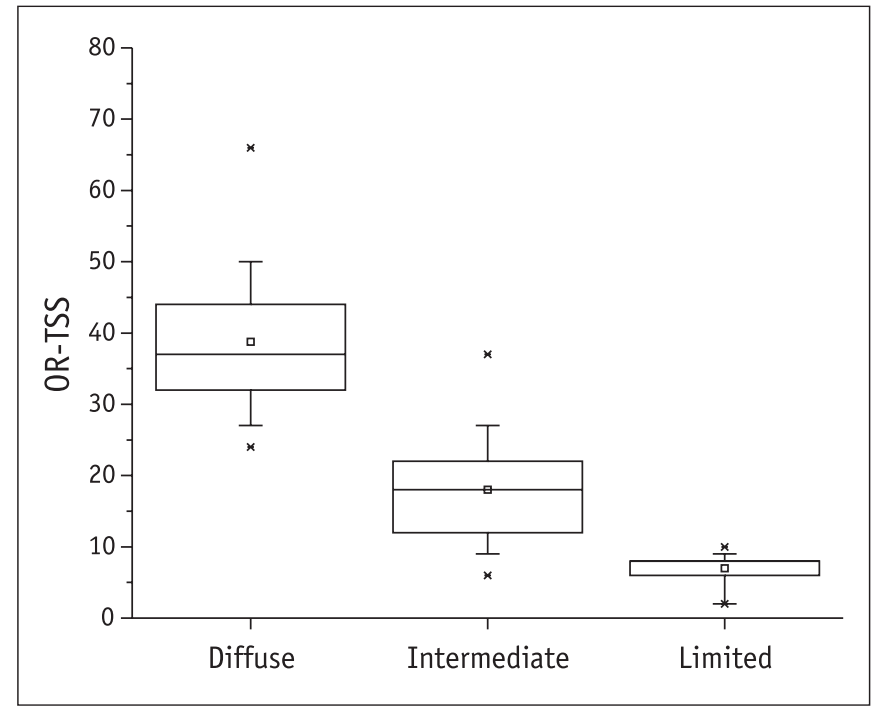

Figura 5 - Escore Cutâneo Total - Método de Rodnan Original (ECT-R0); modelo de três formas clínicas: difusa $(n=12)$, intermediária $(n=34)$ e limitada $(n=10)$; ${ }^{*}<<0,0001$ (teste Mann-Whitney).

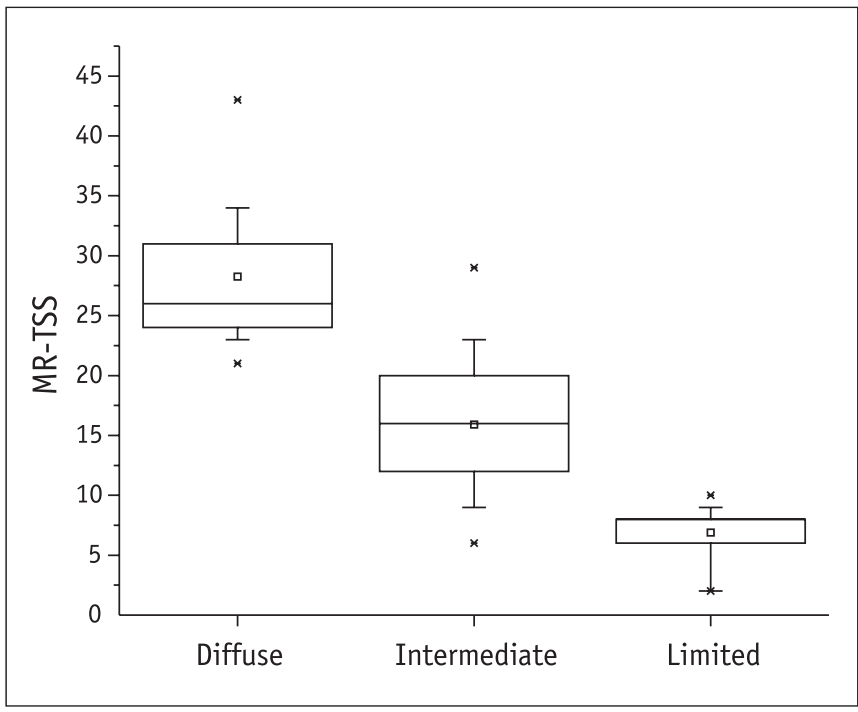

FiguRA 6 - Escore Cutâneo Total - Método de Rodnan Modificado (ECT-RM); modelo de três formas clínicas: difusa $(n=12)$, intermediária $(n=34)$ e limitada $(n=10) ;{ }^{*} p<0,0001$ (teste Mann-Whitney).

Houve associação estatística entre ECT $\leq 20$ e ES limitada (ambos os métodos, no modelo de duas formas clínicas; $p=0.001)$, calcinose (ECT-RM; $p=0.004)$ e anticorpo anticentrômero (ECT-RO, tendência estatística; $p=0.072$ ). $\mathrm{O}$ ECT $>20$ esteve associado à ES difusa (ambos os métodos de ECT, nos dois modelos de ES; $p=0.001)$, distúrbios de pigmentação (ambos os métodos; $p=0.001$ ), acometimento articular $(\mathrm{p}=0.009$ para ECT-RO e $p=0.048$ para ECT-RM), DPR (ECT-RM, tendência estatística; $p=0.054$ ) e anti-topoisomerase I (ECT-RO, tendência estatística; $p=0.080)$.

Foi realizada análise de regressão logística para avaliar as variáveis que influenciaram o ECT. Com respeito ao ECTRO, a análise de regressão logística para variáveis independentes mostrou valores estatisticamente significativos relacionados à forma clínica $(p=0.0001)$ e ao acometimento articular $(p=0.013)$, bem como uma tendência estatística relacionada à calcinose $(p=0.063)$, ao anticorpo anticentrômero $(p=0.077)$ e ao anticorpo anti-topoisomerase $I$ $(p=0.086)$. Quando se utilizou a análise de regressão logística multivariada, foi observado que somente a forma clínica influenciou significativamente o ECT ( $p=0.0001$; RR 99; $95 \%$ IC 15.12 - 648.21). Num segundo modelo, excluindose a variável forma clínica, foi observado que a calcinose $(p=0.0095$; RR 0.151; 95\% IC $0.036-0.630)$ e o acometimento articular $(p=0.0026$; RR 10.18; 95\% IC $2.24-$ 46.25) influenciaram significativamente o ECT.

Com relação aos dados referentes ao ECT-RM, a análise de regressão univariada para variáveis independentes mostrou significativa associação com forma clínica $(p=0.0001)$ e calcinose $(p=0.007)$, bem como uma tendência estatística relacionada ao acometimento articular $(p=0.055)$ e à $D P R$ $(p=0.059)$. Utilizando-se a regressão logística multivariada, foi também observado que somente a variável forma clínica $(p=0.0001 ;$ RR 198.33; IC 95\% 19.18 - 999) influenciou significativamente o ECT, de maneira semelhante à observada com o ECT-RO. Num segundo modelo, excluindo-se a variável forma clínica, também foi observado que a calcinose $(p=0.0011$; RR 0.065 ; 95\% IC $0.013-0.335) \mathrm{e}$ o acometimento articular ( $p=0.0055$; RR 9.09; 95\% IC 1.91 - 43.23) influenciaram significativamente o ECT.

Após um seguimento de cinco anos, houve 20 óbitos (35.7\%). O ECT-RO médio foi de $18.3 \pm 11.7$ (mediana 17.5) para os pacientes vivos e de $24.4 \pm 14.9$ (mediana 20) para os falecidos, enquanto que o ECT-RM médio foi de $15.5 \pm 8.1$ (mediana 15.5) para os pacientes vivos e de $19.6 \pm 9.1$ (mediana 17.5) para os falecidos. Não foi observada associação estatística entre óbito e escores do ECT. As variáveis associadas com óbito foram o acometimento $\operatorname{vascular}(p=0.005)$, esofágico $(p=0.008)$, articular $(p=$ $0.017)$, hipertensão pulmonar $(p=0.025)$ e FAN positivo $(p=0.009)$, com uma tendência estatística relacionada à $\operatorname{DPR}(p=0.066)$. 
A regressão logística multivariada mostrou que o acometimento vascular ( $p=0.0063$; RR 5.25; 95\% IC $1.59-17.27$ ) foi a única variável que influenciou significativamente o óbito; isto indicou que os pacientes com ES e acometimento vascular no início do acompanhamento apresentaram 5.3 vezes mais chances de óbito após seguimento de cinco anos que os pacientes sem esta manifestação.

\section{DISCUSSÃO}

O seguimento da ES ou qualquer outra doença reumática requer medidas estabelecidas para a avaliação do estado atual da doença. A partir do momento que o espessamento da pele é a manifestação mais freqüente e patognomônica da ES, uma maneira útil de acompanhar a evolução dos pacientes esclerodérmicos é o estabelecimento de um "escore cutâneo"(25,26). Embora os escores cutâneos sejam amplamente utilizados para avaliar a evolução da doença em numerosos estudos clínicos, existem dúvidas quanto a sua reprodutibilidade intra e interobservador ${ }^{(19,24)}$.

O ECT original de Rodnan, envolvendo a análise de 26 distintos sítios anatômicos, graduados de 0 (pele normal) a 4 (espessamento extremo), permite a análise global e detalhada do espessamento cutâneo nos pacientes com ES, podendo o médico detectar as menores alterações no envolvimento cutâneo durante o seguimento destes pacientes, mas sua ampla extensão (entre 0 e 104) representa uma causa de grande variabilidade interobservador. $\mathrm{O}$ método ECT original de Rodnan tem sido simplificado pela redução do número de áreas analisadas para $22^{(15)}$ ou $17^{(19,20)}$ e pela restrição do grau de espessamento para 0 a $3^{(15,19,20,24)}$ ou 0 a $2^{(22)}$. O método de ECT de Rodnan modificado mais utilizado inclui a análise de 17 sítios anatômicos graduados de 0 (pele normal) a 3 (espessamento cutâneo intenso); a redução do número de áreas analisadas e da escala de graduação do espessamento cutâneo tende a reduzir a variabilidade intra e interobservador.

Analisando-se e comparando-se os modelos de ES com duas e três formas clínicas no presente estudo, observa-se uma clara distinção entre as formas clínicas limitada e difusa no modelo de duas formas clínicas (ES difusa associada com ECT $>20$, distúrbios de pigmentação, doença pulmonar restritiva e anticorpo antitopoisomerase I, enquanto que a ES limitada esteve associada com ECT $\leq 20$, calcinose e anticorpo anticentrômero). Porém, esta distinção não é tão clara quando se analisa o modelo de ES com três formas clínicas; neste modelo, quando se exclui a extensão do acometimento cutâneo, a distinção entre as formas limitada e intermediária praticamente desaparece.

A subdivisão dos métodos de ECT em ECT baixo $(\leq 20)$ e ECT elevado $(>20)$ pode se tornar útil na detecção dos diferentes padrões de doença. O estudo atual mostrou que um ECT baixo $(\leq 20)$ apresentou correlação estatística com ES limitada e calcinose, com uma tendência estatística relacionada ao anticorpo anticentrômero, enquanto que o ECT elevado (> 20) apresentou associação estatística com ES difusa, distúrbios de pigmentação e acometimento articular, com tendência estatística à associação com DPR e anticorpo antitopoisomerase I. Embora ambos os métodos de ECT apresentassem resultados semelhantes, pode-se interpretar que o ECT-RM representa um método mais prático para o seguimento a longo prazo dos pacientes com ES, em razão de sua menor variabilidade intra e interobservador. Recente estudo falhou ao tentar estabelecer uma relação entre a capacidade funcional e o ECT-RM em 140 pacientes ingleses com $\mathrm{ES}^{(27)}$.

Embora a análise de regressão logística univariada tivesse mostrado que diferentes variáveis (forma clínica, acometimento articular, calcinose, anticorpo anticentrômero, anticorpo antitopoisomerase I) influenciaram o ECT, a regressão logística multivariada estabeleceu que a forma clínica foi a mais importante variável influenciando os escores do ECT, já que um ECT > 20 esteve predominantemente associado com a ES difusa $(90 \%$ com ECT-RO e $85 \%$ com ECT-RM) e um ECT $\leq 20$ esteve associado com a ES limitada (92\% com ECT-RO e 97\% com ECT-RM).

Após um seguimento de cinco anos, verificou-se 35.7\% de óbitos, e esta alta incidência esteve certamente associada com tendências de referência (pacientes com ES mais grave assistidos em um hospital terciário de referência). Apesar das diferentes causas de óbito nos pacientes estudados, a análise de regressão logística multivariada mostrou que o acometimento vascular foi a mais importante variável associada com o óbito, independente dos escores do ECT. A análise das implicações clínicas dos valores do ECT-RM na consulta inicial num extenso estudo multicêntrico avaliando penicilamina em altas doses versus baixas doses na ES mostrou que um ECT-RM inicial $\geq 20$ esteve associado com acometimento cardíaco e foi preditivo de mortalidade e crise renal esclerodérmica nos quatro anos subseqüentes ${ }^{(28)}$. Um estudo britânico analisando a sobrevida de cinco anos em 280 pacientes com ES mostrou que o acometimento cutâneo difuso e os ECTs elevados estiveram associados com uma maior mortalidade na análise univa- 
riada, mas os fatores mais importantes associados com menor sobrevida foram a combinação de proteinúria, velocidade de hemossedimentação elevada e baixa capacidade de difusão de monóxido de carbono $(30 \%)^{(29)}$. Steen e Medsger, analisando a história natural e o estabelecimento de manifestações cutâneas e viscerais graves em 953 pacientes com ES difusa, demonstraram que o acometimento cutâneo grave ocorreu durante os primeiros três anos de doença em $70 \%$ dos casos que desenvolveram este acometimento no seguimento de $10 \operatorname{anos}^{(30)}$. Os mesmos autores demonstraram em estudo recente, analisando 258 pacientes esclerodérmicos com ECT-RM seriados, que a melhora do espessamento cutâneo superior a $25 \%$ (ou, no mínimo, 5 unidades/ano) esteve associada ao melhor prognóstico (90\% em cinco anos e 80\% em 10 anos),

\section{REFERÊNCIAS}

1. Masi AT, Rodnan GP, Medsger TA Jr, et al: Preliminary criteria for the classification of systemic sclerosis (scleroderma). Arthritis Rheum 23: 581-90, 1980.

2. LeRoy EC, Black C, Fleischmajer R, et al: Scleroderma (systemic sclerosis): Classification, subsets and pathogenesis. J Rheumatol 15: 202-5, 1988.

3. Bennett R, Bluestone R, Holt PJL, Bywaters EGL: Survival in scleroderma. Ann Rheum Dis 30: 581-8, 1971.

4. Medsger TA Jr, Masi AT, Rodnan GP, Benedek TG, Robinson H: Survival with systemic sclerosis (scleroderma): A life-table analysis of clinical and demographic factors in 309 patients. Ann Intern Med 75: 369-76, 1971.

5. Arbeitsgruppe der ADF: Klinik der progressiven systemichen sklerodermie (PSS). Multizentrische untersuchungen an 194 patienten. Der Hautartz 37: 320-4, 1986.

6. Giordano M, Valentini G, Migliaresi S, Picillo U, Vatti M: Different antibody patterns and different prognoses in patients with scleroderma with various extent of skin sclerosis. J Rheumatol 13: 911-6, 1986.

7. Barnett AJ, Miller MH, Littlejohn GO: A survival study of patients with scleroderma diagnosed over 30 years (1953-1983): The value of a simple cutaneous classification in the early stages of the disease. J Rheumatol 15: 276-83, 1988.

8. Falanga V, Bucalo B: Use of a durometer to assess skin hardness. J Am Acad Dermatol 29: 47-51, 1993.

9. Aghassi D, Monoson T, Braverman I: Reproducible measurements to quantify cutaneous involvement in scleroderma. Arch Dermatol 131: 1160-6, 1995.

10. Enomoto DNH, Mekkes JR, Bossuyt PMM, Hoekzema R, Bos JD: Quantification of cutaneous sclerosis with a skin elasticity meter in patients with generalized scleroderma. J Am Acad Dermatol 35: 381-7, 1996.

11. Ihn H, Shimozuma M, Fujimoto M, et al: Ultrasound measurement of skin thickness in systemic sclerosis. Br J Rheumatol 34: 535-8, 1995. comparado a um grupo que apresentou piora dos valores de ECT no seguimento (que apresentaram sobrevida de $77 \%$ em cinco anos e $60 \%$ em 10 anos) $)^{(31)}$.

A análise destes resultados indica que o escore cutâneo é um método eficiente e prático no diagnóstico e seguimento da ES, estabelecendo diferentes padrões de doença com implicações prognósticas. O uso periódico do ECT, particularmente do ECT-RM em função de sua maior praticidade, está indicado para o seguimento dos pacientes com ES.

\section{AGRADECIMENTOS}

Os autores agradecem a Helymar da Costa Machado e Cleide Aparecida Moreira Silva a análise estatística deste estudo.

12. Seidenari S, Belletti B, Conti A: A quantitative description of echographic images of sclerotic skin in patients with systemic sclerosis, as assessed by computerized image analysis on $20 \mathrm{MHz}$ B-scan recordings. Acta Derm Venereol (Stockh) 76: 361-4, 1996.

13. Scheja A, Akesson A: Comparison of high frequency $(20 \mathrm{MHz})$ ultrasound and palpation for the assessment of skin involvement in systemic sclerosis (scleroderma). Clin Exp Rheumatol 15: 283-8, 1997.

14. Steen VD, Medsger Jr. TA, Rodnan GP: D-penicillamine therapy in progressive systemic sclerosis (scleroderma). Ann Intern Med 97: 652-9, 1982.

15. Kahaleh MB, Suttany GL, Smith EA, Huffstutter J, Loadholt C, LeRoy EC: A modified scleroderma skin scoring method. Clin Exp Rheumatol 4: 367-9, 1986.

16. Akesson A, Wollheim FA: Organ manifestations in 100 patients with progressive systemic sclerosis: A comparison between the CREST syndrome and diffuse scleroderma. Br J Rheumatol 28: 281-6, 1989.

17. Clements PJ, Lachenbruch PA, Ng SC, Simmons M, Sterz M, Furst DE: Skin score: A semiquantitative measure of cutaneous involvement that improves prediction of prognosis in systemic sclerosis. Arthritis Rheum 33: 1256-63, 1990.

18. Medsger Jr. TA, Steen VD, Ziegler Z, Rodnan GP: The natural history of skin involvement in progressive systemic sclerosis. Arthritis Rheum 23: 720-1, 1980.

19. Brennan P, Silman A, Black C, et al; on behalf of the UK Scleroderma Study Group: Reliability of skin involvement measures in scleroderma. Br J Rheumatol 31: 457-60, 1992.

20. Clements PJ, Lachenbruch PA, Seibold JR, et al: Skin thickness score in systemic sclerosis: An assessment of interobserver variability in 3 independent studies. J Rheumatol 20: 1892-6, 1993.

21. Pope JE, Baron M, Bellamy N, et al: Variability of skin scores and clinical measurements in scleroderma. J Rheumatol 22: 1271-6, 1995.

22. Silman A, Harrison M, Brennan P, and the ad hoc International Group on the Assessment of Disease Outcome in Scleroderma: Is it possible to reduce observer variability in skin score assessment in scleroderma? J Rheumatol 22: 1277-80, 1995. 
23. Clements P, Lachenbruch P, Seibold J, et al: Inter and intraobserver variability of total skin thickness score (modified Rodnan TSS) in systemic sclerosis. J Rheumatol 22: 1281-5, 1995.

24. Furst DE, Clements PJ, Steen VD, et al: The modified Rodnan skin score is an accurate reflection of skin biopsy thickness in systemic sclerosis. J Rheumatol 25: 84-8, 1998.

25. Black CM: Measurement of skin involvement in scleroderma. J Rheumatol 22: 1217-9, 1995.

26. Seibold JR, McCloskey DA; Skin involvement as a relevant outcome measure in clinical trials of systemic sclerosis. Curr Opin Rheumatol 9: 571-5, 1997.

27. Herrick A, Rooney B, Finn J, Silman AJ: Lack of relationship between functional ability and skin score in patients with systemic sclerosis. J Rheumatol 28: 292-5, 2001.
28. Clements PJ, Hurwitz EL, Wong WK, et al: Skin thickness score as a predictor and correlate of outcome in systemic sclerosis. Arthritis Rheum 43: 2445-54, 2000.

29. Bryan C, Knight C, Black CM, Silman AJ: Prediction of five-year survival following presentation with scleroderma: Development of a simple model using three disease factors at first visit. Arthritis Rheum 42: 2660-5, 1999.

30. Steen VD, Medsger TA Jr: Severe organ involvement in systemic sclerosis with diffuse scleroderma. Arthritis Rheum 43: 2437-44, 2000.

31. Steen VD, Medsger TA Jr: Improvement in skin thickening in systemic sclerosis associated with improved survival. Arthritis Rheum 44: 2828-35, 2001. 